\title{
The cardioprotective potential of Tadalafil in myocardial ischemia/reperfusion
}

Najah R. Hadi ${ }^{1}$, Fadhil G. Al-Amran ${ }^{2}$, Ali Abdulzahra Ahmed ${ }^{1}$

\section{Abstract}

The objective of this study is to assess the potential protective effect of Tadalafil on myocardial ischemia reperfusion injury induced by LAD ligation, 28 male rats were randomized into 4 groups (7 rats per group); Sham, rats underwent the same anesthetic and surgical procedure except for LAD ligation; control, rats underwent LAD ligation for 30 minutes and reperfusion for 2 hours; vehicle, rats treated with 10\% DMSO, the Tadalafil solvent 30 minutes before the ligation; Tadalafil group, rats pretreated with Tadalafil $1 \mathrm{mg} / \mathrm{kg}$ i.p 30 minutes before ligation. In control group, as compared with sham, tissue TNF- $\alpha$, IL-6, IL-10, caspase-3 and BAX, plasma cTn-T and serum MDA significantly increased $(P<0.05)$, while serum GSH significantly decreased $(P<0.05)$. Histopathologically, control group showed a significant cardiac injury $(P<0.05)$ compared with sham group. Tadalafil significantly counteracted $(P<0.05)$ the increase of TNF- $\alpha$, IL-6, caspase-3 and BAX and counteracted the increase in plasma cTn-T and serum MDA. Tadalafil produces a significant elevation $(P<0.05)$ in cardiac IL-10 and serum GSH with significant reduction in $(P<0.05)$ cardiac injury. In conclusion, Tadalafil attenuates myocardial I/R injury in male rats through interfering with inflammatory reactions and apoptosis .

Keywords: Tadalafil; LAD ligation; Ischemia/reperfusion; Apoptosis; Inflammatory reactions

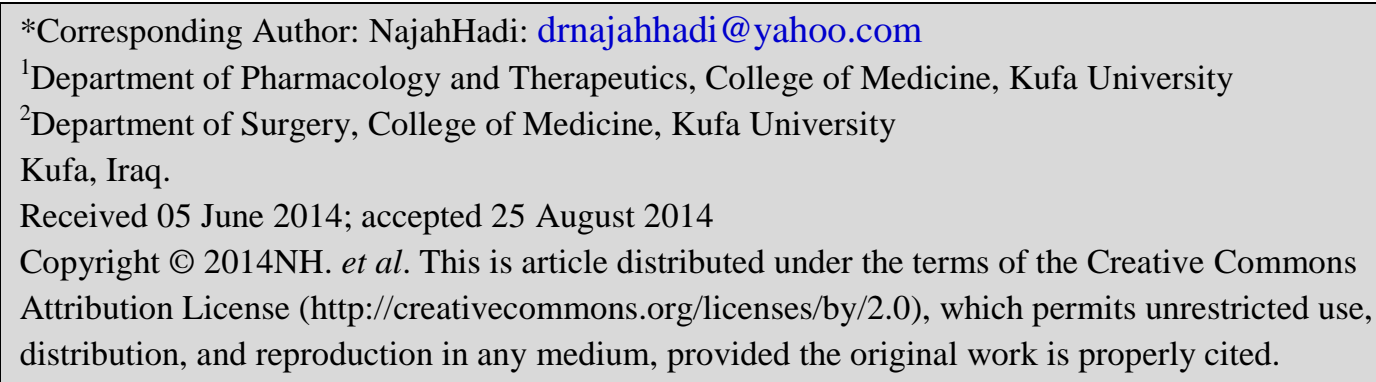




\section{Introduction}

During ischemia to cardiac tissue and as a result of anaerobic glycolysis, $\mathrm{H}+$ will accumulate intracellular leading to a reduction in cell $\mathrm{pH}$ (acidic $\mathrm{pH}$ ) with increased intracellular levels of $\mathrm{Na}+$ and $\mathrm{Ca} 2+$, and on reperfusion of oxygenated blood, there will be a temporary $\mathrm{pH}$ imbalance, associated with an interruption for the electron flow in ETC, and formation of ROS by dysfunctional mitochondria.

This state of imbalance occurs because the acidic environment which was created by myocardial ischemic condition was immediately neutralized extracellular after reperfusion, but intracellular environment remains acidic [1]. Over-production of ROS will alters the electron transport chain that results in dissipating of mitochondrial membrane potential and opening the mPTP and increase calcium ion influx, this will activates pro-apoptotic $\mathrm{BCL}_{2}$ family member protein (BAX) which will cause the formation of MOMP by its binding to the outer membrane of the mitochondria, resulting in efflux of cytochrome-c and other pro-apoptotic factors into the cytosol that leads to the activation of caspase cascade and initiates the apoptosis [2, 3]. Numerous interventions may help to ameliorates IR injury, starting with primary percutaneous coronary intervention (PPCI), ischemic preconditioning, postconditioning, and remote preconditioning [4].

Example of drugs that are represent the pharmacological branch for ameliorating IR are allopurinol as antioxidants, Cyclosporine $\mathrm{A}$ as inhibitors of mitochondrial permeability transition pore (mPTP) opening [5]. PDE-5 enzyme is presents in many tissues including smooth muscles of the corpora cavernosa, platelets, coronary and pulmonary arteries, veins, skeletal muscles, and smooth muscles of bronchi and viscera. Smooth muscle cell relaxation and vasodilatation produced by Tadalafil is achieved by its activity to inhibit the PDE5 enzyme from hydrolyzing cGMP [6].

\section{Method}

\section{Animals}

Pure Tadalafil powder (Sigma, USA),normal saline (KSA) ketamine (Hikma, Jordan), Xylazine (RompunTM, 2\% vials, Bayer AG, Leverkusen, Germany). Rat 
tumor necrosis factor- $\alpha$ (TNF- $\alpha$ ), (IL-6), (IL-10), caspase3, BAX and cTn-T (ELISA) kits were purchased from Biotangusa, USA. Trichloroacetic acid (TCA)MerckGermany, Ethylene diaminetetraacetic acid disodium (EDTA)BDH, UK. Thiobarbituricacid (TBA) Fluka company, Switzerland5, 5-Dithiobis (2nitrobenzoic acid) DTNB Sigma company Ltd. Reduced glutathione Biochemical, USA and Methanol Fluka company, Switzerland. The instruments used in this study were High Intensity Ultrasonic Liquid Processor (Sonics \& materials Inc., USA), Digital Spectrophotometer EMCLAB/Germany, Bio-Elisa Reader, BioTek Instruments, USA and ventilator (Harvard. USA).

After the approval that has been established by the Institutional Animal Care and Use Committee (IACUC) in Kufa university and submission the required applications, a total number of 28 adult male albino rats weighting (180-220 g) were purchased from Animal Resource Center, National Center for Drug Control and Research. They were housed in the animal house of Kufa university/College of Medicine(for one week) in a temperature-controlled $\left(25^{\circ} \pm 1 \mathrm{C}\right)$ room (humidity was kept at (60-65\%) with alternating 12-h light/12-h dark cycles and were allowed free access to water and chow diet until the start of the experimental study.

\section{Experimental design}

After the 1st week of accommodation, the 28 rats were randomly divided into 4 groups (7 rats in each) as follow:

1- (Sham group): Rats underwent the same anesthetic and surgical procedures but without ligation for the LAD.

2-Active control group (induced untreated): rats underwent surgical operation for LAD ligation and they were subjected to $30 \mathrm{~min}$ of ischemia and $120 \mathrm{~min}$ of reperfusion.

3-Vehicle pretreated group: rats were pretreated with DMSO 10\%via intraperitoneal injection 30 minutes before ligation of LAD, then underwent surgical LAD ligation, and subjected to $30 \mathrm{~min}$ of ischemia followed by 120 min of reperfusion.

4-Tadalafil pretreated group: rats of this group take a single I.P injection of Tadalafil in a concentration of $1 \mathrm{mg} / \mathrm{kg}$ dissolved in $10 \%$ DMSO 30 minutes immediately before ligation of LAD, then subjected to surgical LAD ligation with 30 minutes of ischemia followed by120 min of reperfusion [7]. 


\section{Surgical ligation of $L A D$}

All rats were anesthetized by intraperitoneal (IP) injection of $100 \mathrm{mg} / \mathrm{kg}$ ketamine and $5 \mathrm{mg} / \mathrm{kg}$ xylazine [8]. After intubation of the trachea by a $20 \mathrm{G}$ cannula and the endotracheal tube was connected tightly to the ventilation machine. The ventilation rate was fixed from 120-135 breath/minute with tidal volume $20 \mathrm{ml} / \mathrm{kg}$ body weight, with $100 \%$ oxygen. The intercostal muscle layer was gradually cut with micro fine scissors After that the pericardium was opened the left ventricle was visible, the LAD was ligated with an 8-0 prolene suture. The chest wall was closed and at the end of reperfusion time, the animal was re-anesthetized by (IP) mixture of $100 \mathrm{mg} / \mathrm{kg}$ ketamine and $5 \mathrm{mg} / \mathrm{kg}$ xylazine and the chest was re-opened then the right ventricle was punctured with a syringe needle so that about $3 \mathrm{ml}$ of blood was aspirated for later blood analysis. After that, the heart was isolated and divided into 2 pieces, the apical part used for histological examination and the basal was used for measuring the tissue parameters .

Blood sampling for measurement of plasma $c T n-T$, serum MDA and serum reduced GSH

At the end of experiment, about 2-3 $\mathrm{ml}$ of blood was collected by disposable syringe from the heart of each rat via cardiac puncture. The first half was placed immediately in a tube containing disodium EDTA $(22 \mathrm{mg} / \mathrm{ml})$ as anticoagulant and mixed thoroughly then centrifuged at $3000 \mathrm{rpm}$ for $15 \mathrm{~min}$ then the supernatant was used for determination of plasma cTn-T level, while the remaining blood was allowed to clot in an ordinary tube at $37^{\circ} \mathrm{C}$ then it was centrifuged at $3000 \mathrm{rpm}$ for 15 minutes then the supernatant was used for the determination of serum MDA and GSH levels.

\section{Tissue preparation for $T N F-\alpha, I L-6, I L-10$, caspase 3 and BAX measurements}

The heart of each rat which was excised at the end of the reperfusion time, was washed with ice cold saline to exclude clots, any remaining parts of the atria were removed also, then homogenization for the cardiac tissue was done with a high intensity ultrasonic liquid processor in 1:10 (w/v) phosphate buffered saline that contained 1\% Triton X-100 and a protease inhibitor cocktail [9]. Then tissue homogenate was centrifuged at $14000 \mathrm{rpm}$ for $20 \mathrm{~min}$ at $4^{\circ} \mathrm{C}$. After this step, the collection of the tissue homogenate supernatant was done in order to detect the levels of TNF- $\alpha$, IL-6, IL-10, caspase 3 and BAX by the ELISA technique with a commercially available ELISA kit ( literature of kit by Biotangusa, USA.) according to the manufacturer's instructions. 


\section{Tissue Preparation for Histopathology}

The myocardial tissue that reserved for histopathological study was fixed in $10 \%$ formalin and embedded in a block of paraffin. The $5 \mu \mathrm{m}$ sections which were cut from each block were stained by hematoxylin and eosin (H\&E) after fixation. Damage scores were evaluated according to the following morphological criteria that have been used to evaluate the histopathological damage [10] as follow:

1- Score 0, no damage.

2- Score 1 (mild), interstitial edema and focal necrosis.

3- Score 2 (moderate), diffuse myocardial cell swelling and necrosis.

4- Score 3 (severe), necrosis with the presence of contraction bands, neutrophil infiltration and the capillaries were compressed.

5- Score 4 (highly severe), widespread necrosis with the presence of contraction bands, neutrophil infiltration, capillaries compressing and hemorrhage.

\section{Statistical analyses}

Data were expressed as mean \pm SEM. An expert statistical advice was considered for data analysis which were aided by computer. Statistical analysis were done using SPSS version 20.0 computer software (Statistical Package for Social Science). ANOVA (analysis of variance) had been used for measurement (numerical data). Mann-Whitney test had been used for myocardial damage score. P value $<0.05$ regarded as significant.

\section{Results}
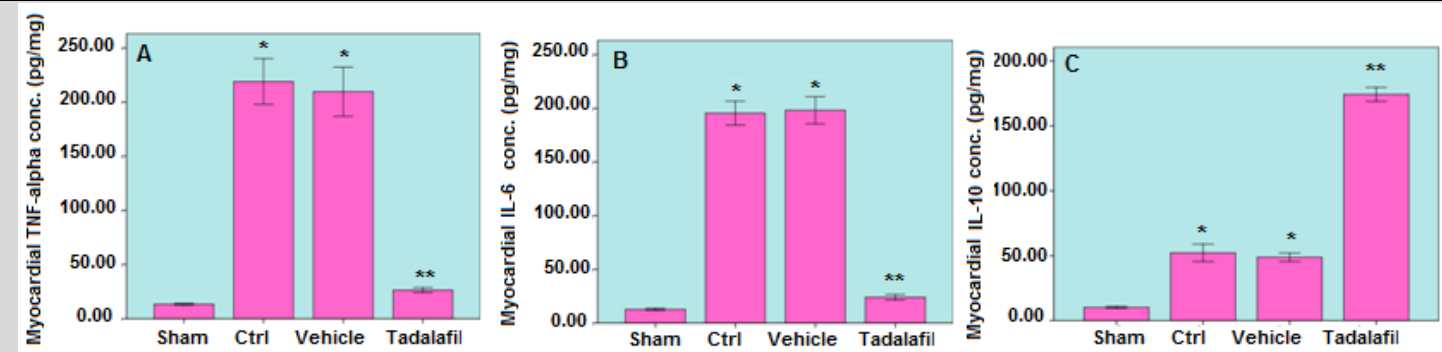

Figure 1.

The mean of myocardial cytokines level A) TNF- $\alpha$, B) IL-6, C) IL-10 (pg/mg) in the four experimental groups at the end of the experiment. ${ }^{*} P<0.05$ vs. sham group $;^{* *} P<0.05$ vs. Ctrl vehicle group. 

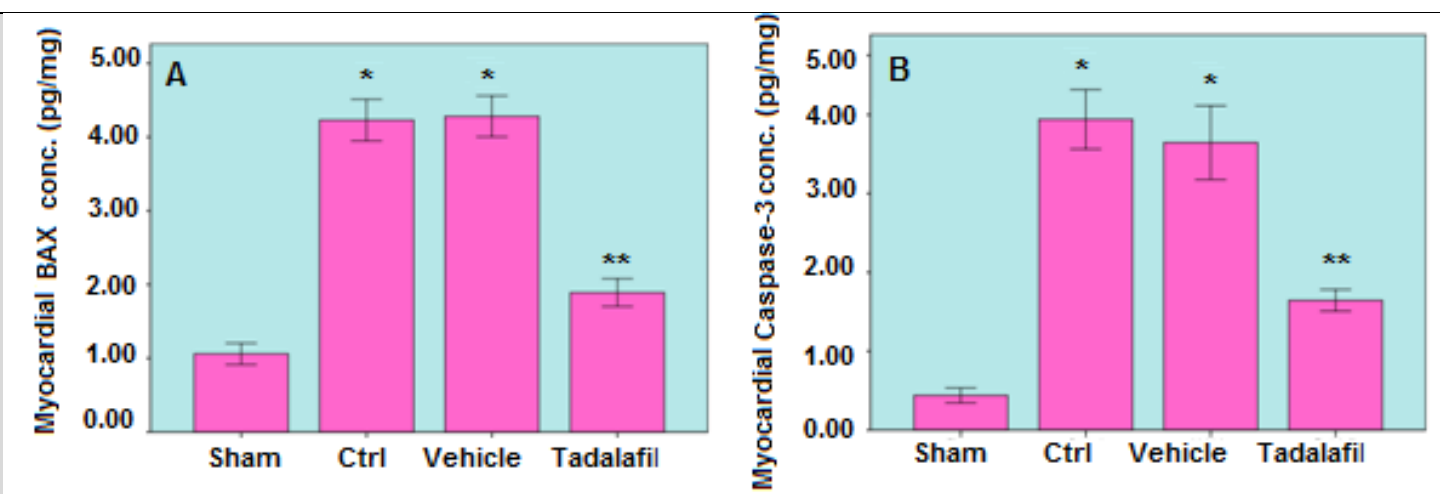

Figure 2.

The myocardial mean of: A) BAX and B) Caspase-3 (pg/mg) in the four experimental groups at the end of the experiment. ${ }^{*} P<0.05 v s$. sham group, ${ }^{* * *} P<0.05$ vs. Ctrl vehicle group.

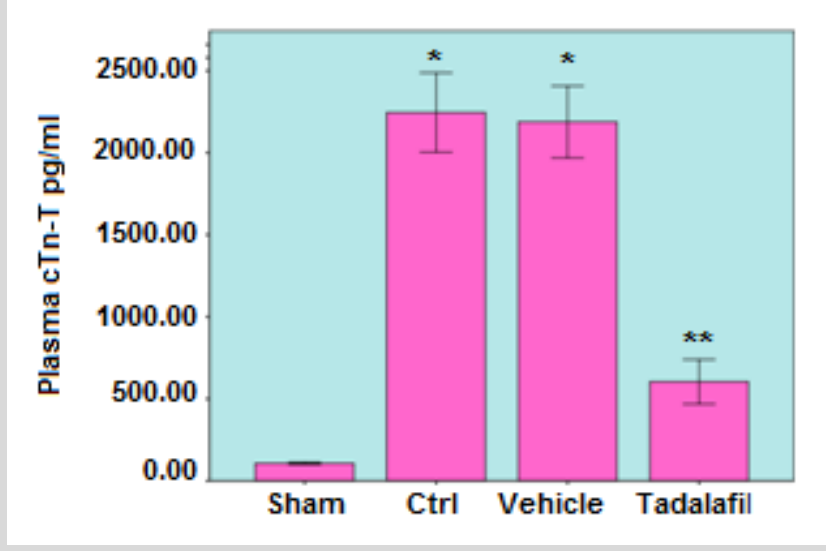

Figure 3.

The mean of plasma cTn-T level $(\mathrm{pg} / \mathrm{ml})$ in the four experimental groups at the end of the experiment. $P<0.05$ vs. sham group, ${ }^{* *} P<0.05$ vs. Ctrl vehicle group. 

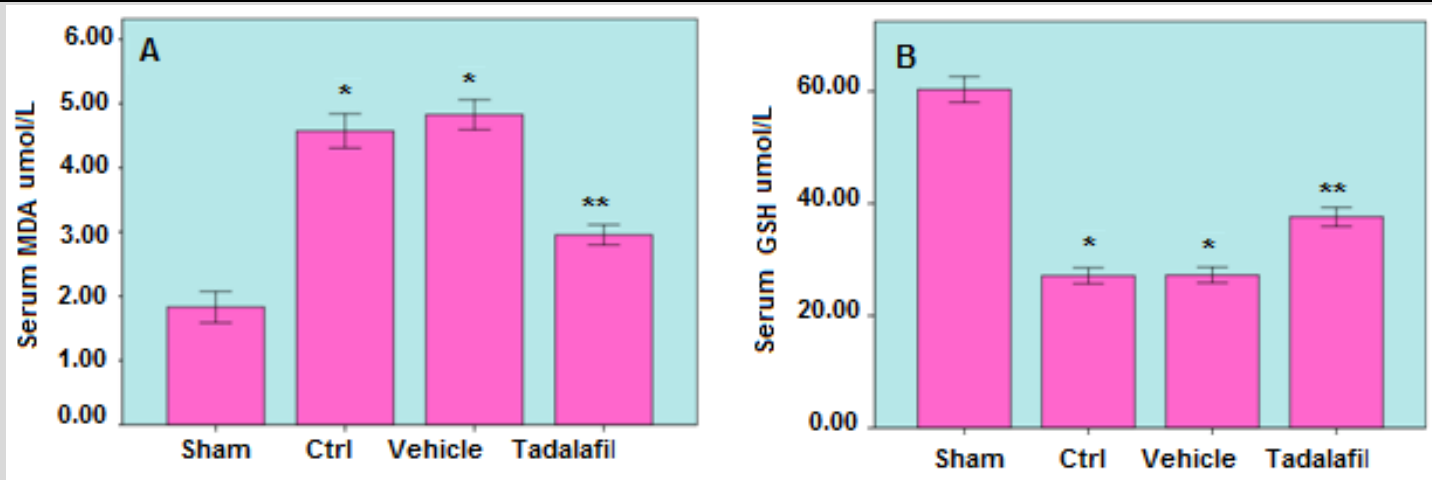

\section{Figure 4.}

The myocardial mean of: A) MDA and B) GSH ( $\mu \mathrm{mol} / \mathrm{L})$ in the four experimental groups at the end of the experiment. ${ }^{*} P<0.05 v s$. sham group, ${ }^{* *} P<0.05 v s$. Ctrl vehicle group.

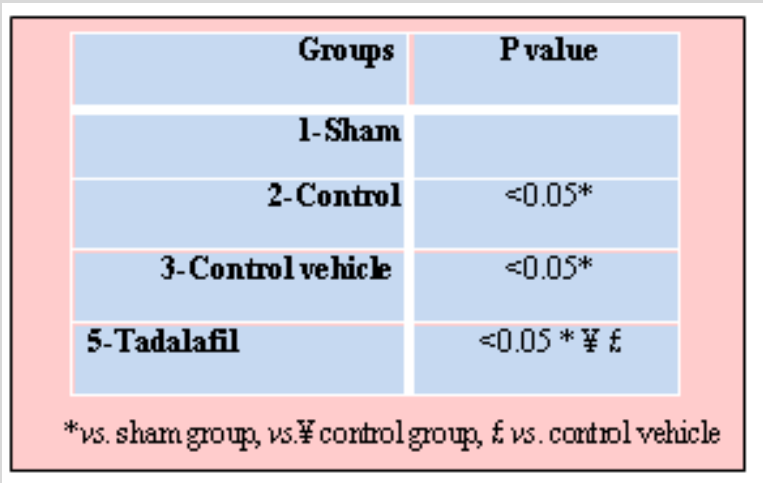

Table 1.

Comparison according to Mann-Whitney test for scoring regarding histopathological changes.

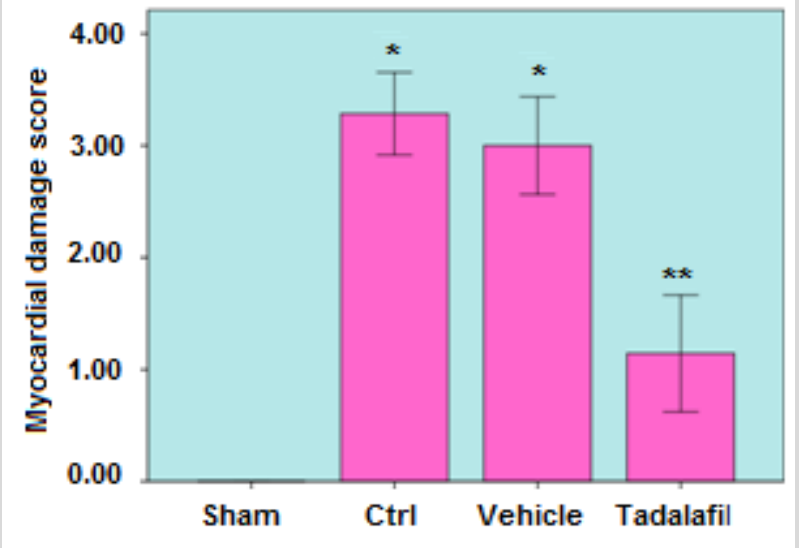

Figure 4.

Component bar chart shows the relative frequency of different histopathological grading of abnormal heart changes among the four experimental groups. 

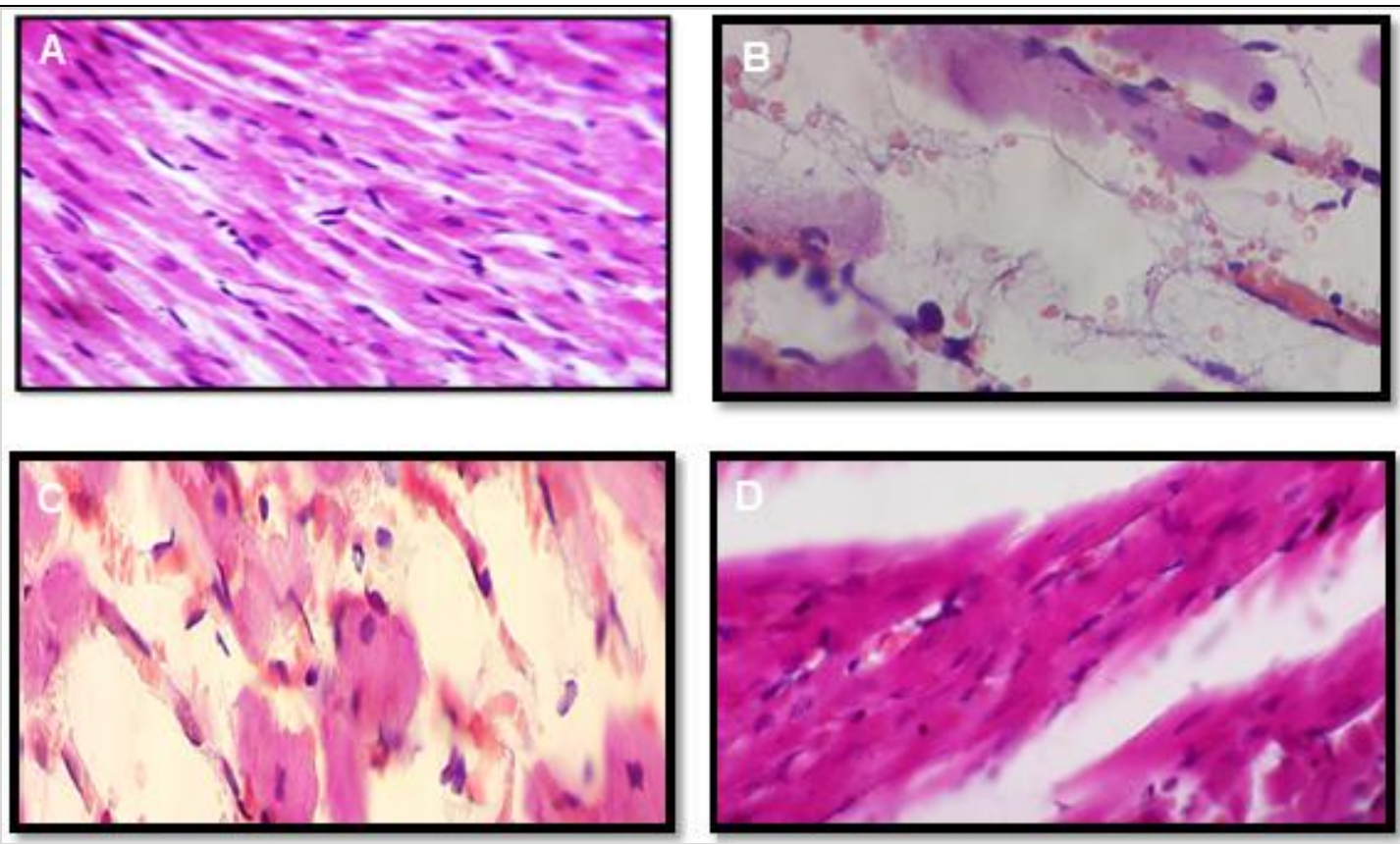

Figure 5.

Representative photomicrograph of a section of the heart tissue section stained with Haematoxylin and Eosin (X 40). A)The sham group shows normal architecture. B) The control group showing sever hemorrhage and extravasation of $\mathrm{RBC}$, presence of sever interstitial edema, presence of neutrophil infiltration and necrosis. C) The vehicle group showing sever hemorrhage and extravasation of RBC, presence of sever interstitial edema, presence of neutrophil infiltration and necrosis. D) The Tadalafil pretreated group showing near normal cardiac tissue with absence of edema, absence of neutrophil infiltration, absence of necrosis, and only congested capillary structure.

Results revealed a significant increase $(P<0.05)$ in TNF- $\alpha$ and IL-6 cardiac tissue levels in the active control group as compared with the sham group, while in the Tadalafil pretreated group, Tadalafil produce a significant reduction $(P<0.05)$ in the TNF- $\alpha$ and IL- 6 cardiac tissue levels as compared with the active control group and vehicle group as shown in figures1 A, B.

Further, results revealed a significant increase $(\mathrm{P}<0.05)$ in IL-10 cardiac tissue level in the active control group as compared with the sham group, while in the Tadalafil pretreated group, Tadalafil produce a significant elevation $(\mathrm{P}<0.05)$ in the (IL-10) cardiac tissue level as compared with all other groups (sham group, the active control group and vehicle group as shown in figure $1 \mathrm{C}$. The caspase-3 and BAX cardiac tissue levels are increased in the active control group as compared with the sham group, while in the Tadalafil pretreated group, Tadalafil produce a significant 
reduction $(P<0.05)$ in the caspase-3 and BAX cardiac tissue levels as compared with the active control group and vehicle group as shown in figures $2 \mathrm{~A}, \mathrm{~B}$.

The (cTn-T) plasma level in the active control group is as increased compared with the sham group, while in the Tadalafil pretreated group, Tadalafil produce a significant reduction $(P<0.05)$ in the $(\mathrm{cTn}-\mathrm{T})$ plasma level as compared with the active control group and vehicle group as shown in figure 3.

The serum level of MDA is elevated in the active control group as compared with the sham group, while in the Tadalafil pretreated group, Tadalafil produce a significant reduction $(P<0.05)$ in MDA serum level as compared with the active control group and vehicle group. Regarding GSH, study revealed a significant decrease $(P<0.05)$ in the serum level of GSH in the active control group as compared with the sham group, while in the Tadalafil pretreated group, Tadalafil produce a significant increase $(P<0.05)$ in GSH serum level as compared with the active control group and vehicle group as shown in and figures $4 \mathrm{~A}, \mathrm{~B}$.

Examination of a cross section from the active control group revealed a significant cardiac tissue injury $(P<0.05)$ compared with the sham group, and this injury was showing sever hemorrhage and extravasation of RBC, presence of sever interstitial edema, presence of neutrophil infiltration and necrosis on the contrast of the cross section of the sham group which showed a 100\% normal structure regarding cardiac tissue. Treatment of rats with Tadalafil significantly decrease $(P<0.05)$ the injury of cardiac tissue and cross section from this group showed near normal cardiac tissue with absence of edema, absence of neutrophil infiltration, absence of necrosis, and only congested capillary structure while there was no significant difference between the control and vehicle group as shown in table 1 and figures $5 \mathrm{~A}, \mathrm{~B}, \mathrm{C}, \mathrm{D}$.

\section{Discussion}

On reperfusion, the deleterious conversion of reversible ischemia into irreversible cardiomyocyte death or remodeling occurs through a complex cascade of reactions that involves increased levels of ROS, formation of inflammatory cytokineslike TNF$\alpha$, IL-6, IL-10, pro-apoptotic caspase-3 and Bax proteins, vascular endothelium dysfunction and inflammatory responses that mediated by cells of the immune system like activated neutrophils $[11,12]$. 
Pretreatment with tadalafil before induction of myocardial ischemia produced a significant reduction $(\mathrm{P}<0.05)$ in the myocardial tissue levels of pro-inflammatory cytokines (TNF- $\alpha$, IL-6 ), with the significant elevation $(\mathrm{P}<0.05)$ in the level of antiinflammatory cytokine IL-10 compared to the control group and vehicle group. Varma et al (2012) demonstrated that pretreatment with Tadalafil significantly reduce the level of TNF- $\alpha$ with a paradoxical significant increase in the level of IL-10 accompanied by a decrease of IL-6 level after reperfusion of ischemic heart in a diabetic mice model [13]. Furthermore, Wang et al (2013) clarified that the significant reduction of TNF- $\alpha$ and IL-6 tissue levels was strongly associated with the cardioprotective pathway after reperfusion in a rat model of ischemia reperfusion injury [14].

The level of caspase- 3 and BAX in cardiac tissue was significantly decreased $(P<0.05)$ in the Tadalafil pretreated group compared to the control group and vehicle group. To best of our knowledge, there is no study measured the effect of Tadalafil on Caspase-3 and Bax in myocardial ischemia reperfusion injury, however,Varma et al (2012) also showed that apoptosis level was reduced in Tadalafil pretreated isolated mice cardiomyocytes as appears through the reduction of TUNEL positive cardiomyocyte number [13]. Baek et al (2011) showed that the anti-apoptotic activity of Tadalafil in the hippocampus of maternal-separated rat pups appears through the significant reduction of the expression of active caspase-3 detected by TUNEL test [15].

Ko et al (2009) proved that pretreatment with Tadalafil suppress apoptosis through significant reduction of caspase- 3 expression induced by cerebral ischemia in gerbils model [16]. Koka et al (2010) clarified that the anti-apoptotic effect of Tadalafil on cardiac tissue in a mice model appears through its significant elevation for the antiapoptotic bcl2 protein [17]. Whelan et al (2012) clarified that the deletion of Bax, the pro-apoptotic protein results in a dramatic reduction in necrosis and resistance to reperfusion injury in an in vivo model of myocardial infarction in Bax/Bak knockout mice [18].

\section{Effect of Tadalafil on cTn-T level}

The cTn-T plasma level of Tadalafil pretreated group was significantly decreased $(\mathrm{P}<0.05)$ compared to the control group and the vehicle group. To best of our knowledge, there is no study measured the effect of Tadalafil on cTnT in myocardial ischemia reperfusion injury, however because Tadalafil is a member of selective 
PDE5 enzyme inhibitor family, so its typically accepted that its activity is similar to that of other members, as sildenafil effect in the study of Hassan et al (2005) which clarified that the use of sildenafil significantly reduce blood cTn-T level in rat model of induced myocardial hypertrophy indicating the cardio-protective effect of PDE5 enzyme inhibitors that includes Tadalafil [19].

\section{Effect of Tadalafil on MDA and reduced GSH level}

There was a significant decrease $(P<0.05)$ in serum MDA level with a significant elevation $(P<0.05)$ of GSH serum level in the tadalafil pretreated group compared to the active and control vehicle group. Koka et al (2010) clarified that Tadalafil reduce the oxidative stress in mice cardiomyocytes through its ability to decrease MDA level and enhancing the mitochondrial anti-oxidant activity in a Doxorubicin-Induced Cardiomyopathy model [17].

Arikan et al (2010) proved that the protective effect of pretreatment with Tadalafil in reducing the reperfusion injury of ischemic rat ovary was accompanied by a significant reduction of the Malondialdehyde (MDA) level, the tissue injury parameter that produced from lipid peroxidation as a result of oxidative stress [20]. Serarslan et al (2010) showed that Tadalafil protective effect in decreasing the spinal cord injury in a rat model was associated with significant reduction in MDA level with paradoxical significant elevation of anti-oxidant enzymatic activity as GSH-Px and sSOD [21].

Koka et a $l(2012)$ demonstrated that treatment of diabetic mice with Tadalafil reduce the cardio-vascular damage due to reduction in the formation of ROS by the significant reduction in the ratio of GSSG/GSH indicating the increased level of reduced GSH and the reduction in oxidative stress [22], furthermore, Koka et al (2013) demonstrated that Tadalafil have a protective effect against myocardial ischemia reperfusion injury in diabetic mice through the significant reduction in the MDA level accompanied by the significant enhancement of the reduced GSH level confirming its anti-oxidative stress activity represented by significant reduction of ROS through the marked inhibition of activity and expression of NADPH oxidase enzyme [7]. Gulati et al (2013) clarified that the neuro-protective effect of Tadalafil against cerebral ischemia reperfusion injury associated with significant elevation in the level of reduced GSH [23].

Treatment of rats with Tadalafil significantly reduce cardiac injury $(P<0.05)$ as compared with active control group and vehicle group. The scores of the control group 
demonstrates a $28.5 \%$ with highly severe myocardial injury and $71.5 \%$ with sever myocardial injury, while the score of Tadalafil treated group were $14.25 \%$ of the group had no damage, $57.25 \%$ had mild cardiac injury and $28.5 \%$ had moderate cardiac injury. Sesti et al. (2007) proved that Tadalafil significantly reduce the infarct size after reperfusion of ischemic myocardium in male rats [24]. Salloum et al (2009) also proved the cardio-protective effect of Tadalafil against myocardial ischemia reperfusion injury through its ability to significantly reduce the infarct size in cardiac tissue in mice model [25].

In conclusion: It can be concluded that pretreatment with Tadalafil modulates myocardial ischemia reperfusion injury via interfering with inflammatory, oxidative pathways and apoptosis.

\section{Competing interests}

The authors declare that there is no conflict of interest.

\section{Author Contributions}

All authors wrote, read and approved the final manuscript.

\section{References}

1. Lee JA, Allen DG. Mechanisms of acute ischemic contractile failure of the heart. Role of intracellular calcium. J Clin Invest 1991;88(2):361-7.

2. Lemasters JJ, Qian T, He L, Kim JS, Elmore SP, Cascio WE, et al. Role of mitochondrial inner membrane permeabilization in necrotic cell death, apoptosis, and autophagy. Antioxid Redox Signal 2002;4(5):769-81.

3. Di Lisa F, Menabo R, Canton M, Barile M, Bernardi P. Opening of the mitochondrial permeability transition pore causes depletion of mitochondrial and cytosolic NAD+ and is a causative event in the death of myocytes in postischemic reperfusion of the heart. J Biol Chem 2001;276(4):2571-5.

4. Bahde R, Spiegel HU. Hepatic ischaemia-reperfusion injury from bench to bedside. Br J Surg 2010;97(10):1461-75

5. Onishi A, Miyamae M, Kaneda K, Kotani J, Figueredo VM. Direct evidence for inhibition of mitochondrial permeability transition pore opening by sevoflurane preconditioning in cardiomyocytes: comparison with cyclosporine A. Eur J Pharmacol 2012;675(1-3):40-6. 
6. Morelli A, Sarchielli E, Comeglio P, Filippi S, Mancina R, Gacci M, et al. Phosphodiesterase type 5 expression in human and rat lower urinary tract tissues and the effect of tadalafil on prostate gland oxygenation in spontaneously hypertensive rats. J Sex Med 2011;8(10):2746-60.

7. Koka S, Das A, Salloum FN, Kukreja RC. Phosphodiesterase-5 inhibitor tadalafil attenuates oxidative stress and protects against myocardial ischemia/reperfusion injury in type 2 diabetic mice. Free Radic Biol Med 2013;60:80-8.

8. Hadi NR, Yuosif FG, Yousif M, Jaen KK. Both castration and goserelin acetate ameliorate myocardial ischemia reperfusion injury and apoptosis in male rats. ISRN Pharmacol 2014; 2014:206951.

9. Zhang M, Xu YJ, Saini HK, Turan B, Liu PP, Dhalla NS. Pentoxifylline attenuates cardiac dysfunction and reduces TNF-alpha level in ischemic-reperfused heart. Am J Physiol Heart Circ Physiol 2005;289(2):H832-9.

10. Zingarelli B, Salzman AL, Szabo C. Genetic disruption of poly (ADP-ribose) synthetase inhibits the expression of P-selectin and intercellular adhesion molecule-1 in myocardial ischemia/reperfusion injury. Circ Res 1998;83(1):85-94.

11. Austin EW, Yousif NG, Ao L, Cleveland JC, Fullerton DA, Meng X. Ghrelin reduces myocardial injury following global ischemia and reperfusion via suppression of myocardial inflammatory response. American Journal of BioMedicine 2013;1: 38-48.

12. Hochhauser E, Kivity S, Offen D, Maulik N, Otani H, Barhum Y, et al. Bax ablation protects against myocardial ischemia-reperfusion injury in transgenic mice. Am J Physiol Heart Circ Physiol 2003;284(6):H2351-9.

13. Varma A, Das A, Hoke NN, Durrant DE, Salloum FN, Kukreja RC. Antiinflammatory and cardioprotective effects of tadalafil in diabetic mice. PLoS One 2012;7(9):e45243.

14. Wang Y, Zhang ZZ, Wu Y, Zhan J, He XH, Wang YL. Honokiol protects rat hearts against myocardial ischemia reperfusion injury by reducing oxidative stress and inflammation. Exp Ther Med 2013;5(1):315-9.

15. Baek SB, Bahn G, Moon SJ, Lee J, Kim KH, Ko IG, et al. The phosphodiesterase type-5 inhibitor, tadalafil, improves depressive symptoms, ameliorates memory impairment, as well as suppresses apoptosis and enhances cell proliferation in the hippocampus of maternal-separated rat pups. Neurosci Lett 2011;488(1):26-30.

16. Ko IG, Shin MS, Kim BK, Kim SE, Sung YH, Kim TS, et al. Tadalafil improves short-term memory by suppressing ischemia-induced apoptosis of hippocampal neuronal cells in gerbils. Pharmacol Biochem Behav 2009;91(4):629-35.

17. Koka S, Das A, Zhu SG, Durrant D, Xi L, Kukreja RC. Long-acting phosphodiesterase-5 inhibitor tadalafil attenuates doxorubicin-induced cardiomyopathy without interfering with chemotherapeutic effect. J Pharmacol Exp Ther 2010;334(3):1023-30.

18. Whelan RS, Konstantinidis K, Wei AC, Chen Y, Reyna DE, Jha S, et al. Bax regulates primary necrosis through mitochondrial dynamics. Proc Natl Acad Sci USA 2012;109(17):6566-71. 
19. Hassan MA, Ketat AF. Sildenafil citrate increases myocardial cGMP content in rat heart, decreases its hypertrophic response to isoproterenol and decreases myocardial leak of creatine kinase and troponin T. BMC Pharmacol 2005;5:10.

20. Arikan DC, Bakan V, Kurutas EB, Sayar H, Coskun A. Protective effect of tadalafil on ischemia/reperfusion injury of rat ovary. J Pediatr Surg 2010;45(11):2203-9.

21. Serarslan Y, Yonden Z, Ozgiray E, Oktar S, Guven EO, Sogut S, et al. Protective effects of tadalafil on experimental spinal cord injury in rats. J Clin Neurosci 2010;17(3):349-52.

22. Koka S, Xi L, Kukreja RC. Chronic treatment with long acting phosphodiesterase-5 inhibitor tadalafil alters proteomic changes associated with cytoskeletal rearrangement and redox regulation in Type 2 diabetic hearts. Basic Res Cardiol 2012;107(2):249.

23. Gulati P, Singh N. Neuroprotective effect of tadalafil, a PDE-5 inhibitor, and its modulation by L-NAME in mouse model of ischemia-reperfusion injury. $J$ Surg Res 2014;186(1):475-83.

24. Sesti C, Florio V, Johnson EG, Kloner RA. The phosphodiesterase-5 inhibitor tadalafil reduces myocardial infarct size. Int J Impot Res 2007;19(1):55-61.

25. 25 Salloum FN, Chau VQ, Hoke NN, Abbate A, Varma A, Ockaili RA, et al. Phosphodiesterase-5 inhibitor, tadalafil, protects against myocardial ischemia/reperfusion through protein-kinase g-dependent generation of hydrogen sulfide. Circulation 2009; (11 Suppl):S31-6. 